\title{
Research on the Somatosensory Command System on Stage Performance Effect
}

\author{
Yang Chen \\ Huanghe Science and Technology College \\ Zhengzhou, China
}

\begin{abstract}
With the rapid updating of hardware technology, the somatosensory device with multi-sensing mode is gradually used in human-computer interaction interface. The existing opera performance can not meet the need of somatosensory equipment input, especially in the digital creation field, thus it is one of the key technologies to research the somatosensorysupporting remote virtual command system.
\end{abstract}

Keywords-somatosensory equipment; human-computer interaction; opera performance; remote command system

\section{INTRODUCTION}

With the rapid updating of hardware technology, the somatosensory device with multi-sensing mode is gradually used in human-computer interaction interface. The existing opera performance can not meet the need of somatosensory equipment input, especially in the digital creation field, thus it is one of the key technologies to research the somatosensorysupporting remote virtual command system, besides, how to realize the perfect combination of advanced technology and culture $\&$ art, and break through the barriers of discipline, field and industry have become urgent problems which affect our cultural industry's rapid development. Make full use of the current advanced technology and apply to the integrated innovation of cultural service, start from the technologies such as cultural and creative industry content, intelligent creativity, virtual world, efficient real-time interaction and so on, integrate and optimize the cultural sustainable industrial development capacity of the traditional manual operation and man power production, have proved to be the only way for sustainable development, and the form of emerging industry, the digital stage technology industry has emerged.

\section{SYSTEM OVERVIEW}

Remote virtual command system mainly completes analysis and processing of data collected by Kinect motion acquisition equipment, the data collected here is mainly distinction and processing of human bones and baton bones. The captured bones can be differentiated and rendered and output separately by analyzing the obtained data.

The system firstly collect the continuous images of the movement of the conductor by Kinect motion capture device, analyzes the collected images, and calculates the position, direction and speed of the conductor and the baton in his/her hands. And then it transmits the data to the underlying graphics rendering engine by the remote communication system, through calculation of the rendering engine, it transmits to the video output device, and reproduces the motion of the conductor and baton in the scene with the engine's default Cartesian coordinate system "Fig. 1".

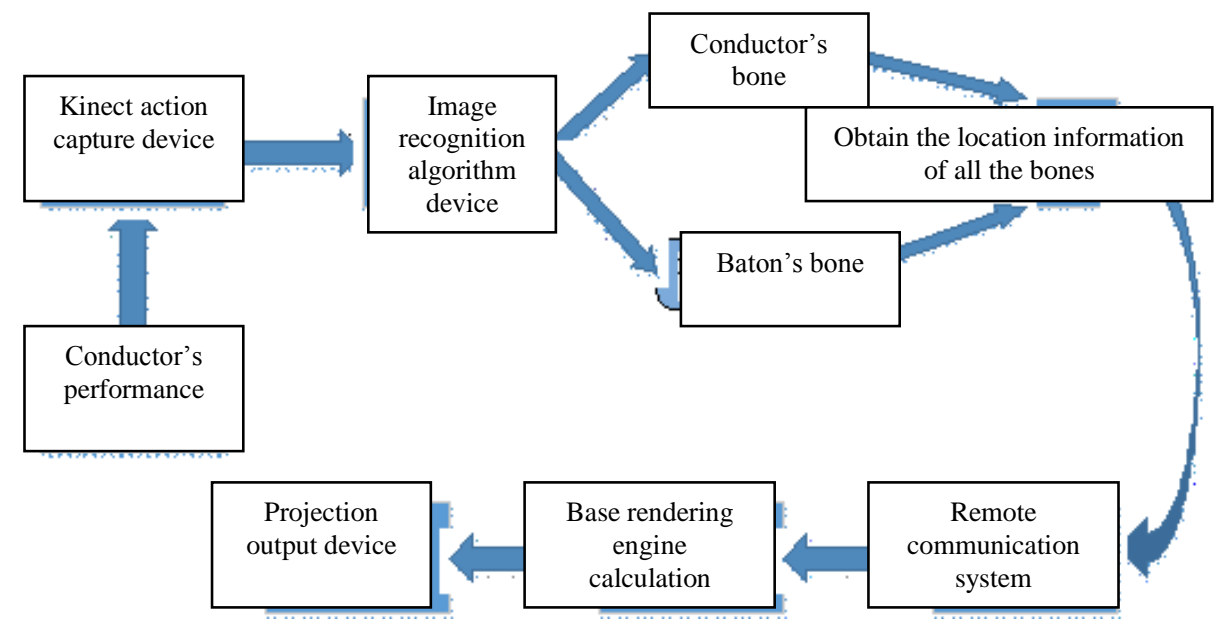

Fig. 1. Flow chart of conducting motion capture and data transmission. 


\section{CAPTURE THE MOTION CAPTURE DATA WITH KINECT}

Kinect sensor is a device whose appearance is similar to network camera. Kinect has three lenses, whose middle lens is RGB color camera, left and right lens are 3D structured light depth sensors constituted by infrared emitter and infrared CMOS camera. Kinect is also equipped with focus tracing technology, and the base motor will rotate following the movement of the focused object. Kinect also has built-in array microphone, and the multiple groups of microphones record sound at the same time, and eliminates the noise after comparing.

Kinect has $1080 \mathrm{p}$ video sensor, can get clearer image and video information. The maximum resolution that the depth sensor can support allows to capture more detail, such as finger motion and limb positioning. Kinect can process data up to 2GB per second. Microsoft replaces the existing measurement method of "light measurement" with the method of reading the depth to "calculate flight time", making Kinect faster and more accurate. This method is based on the most accurate time calculation principle of a single photon bouncing from an object or a human body. In this project, we capture actors' motions in real time with Kinect to realize the motion capture of the human model for construction of model base and motion base, and the real-time interactive rehearsal manipulation, interacting with other object roles in the stage scene, triggering the set response of other objects or roles, making the digital performance more realistic, bringing more shocking visual experience to audience by rendering the visual effect and adding special effects of thunder. As shown in the figure, the somatosensory interactive control of the human model is achieved through the control structure flow setting in the engine and Kinect operation interface "Fig. 2". Kinect data can introduce real-time data into the engine and interact with the objects in $3 \mathrm{~d}$ engine, and can also scan a lower-precision stage props and human models and store into the model base.

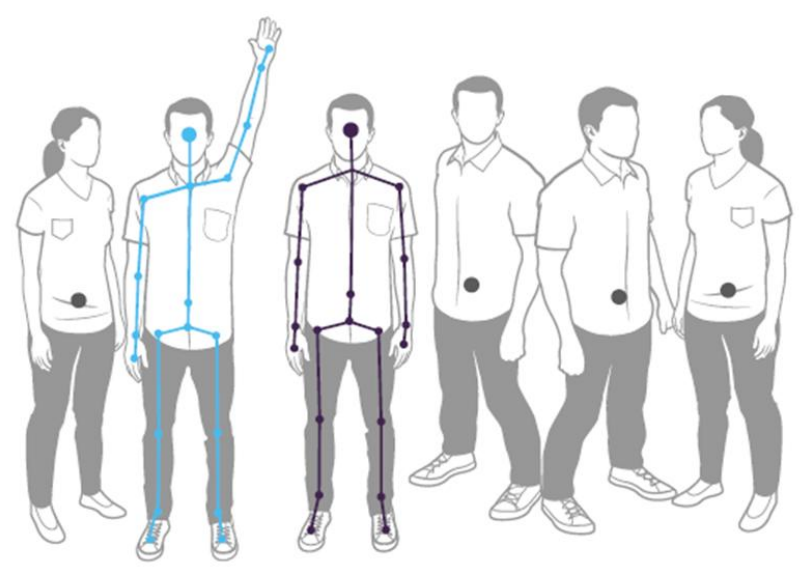

Fig. 2. Somatosensory motion recognition.

\section{HUMAN BONE RECOGNITION}

In Kinect, a skeleton is represented by 20 joints, and the detail (as shown in "Fig. 3") can be seen. When entering the vision scope of Kinect, Kinect can find the location of the 20 joints of the human body, the location is represented by $(\mathrm{x}, \mathrm{y}, \mathrm{z})$ coordinates. In this way, when Kinect does a lot of complex motions before, because thee is still a great relationship between the human motion and the location of these joints, when the computer receives the data, it is helpful to understand the performers' motions.

The position of each joint of the player is represented by ( $\mathrm{x}$, $\mathrm{y}, \mathrm{z})$ coordinates. Unlike the depth image spatial coordinates, these coordinates' unit is meter. The coordinate axes $\mathrm{x}, \mathrm{y}, \mathrm{z}$ are the spatial $\mathrm{x}, \mathrm{y}, \mathrm{z}$ axes of the depth sensor entity. This coordinate system is right-handed spiral, Kinect sensor is at the origin, and z-axis is in the same direction as Kinect sensor. The positive half $y$-axis extends upward and the positive half $x$-axis (from the visual angle of Kinect sensor) extends to the left, as shown in the following figure.

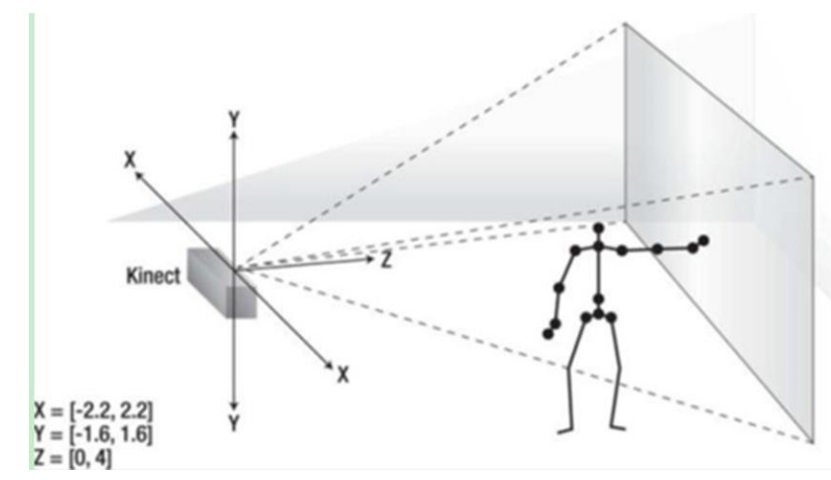

Fig. 3. Kinect skeleton display.

\section{BATON BONE EXTRACTION}

The module firstly obtains Kinect's depth diagram, and then tracks the position of the center point of the hand with NITE base. After tracking and detecting the position of the hand point, the depth map is segmented according to the $\mathrm{Z}$ coordinate of the center point of the hand, and then the hand center point is projected to the two-dimensional space to be divided again according to the size of the hand.

A part containing arm, hand and baton will be obtained thus, and the characteristics of Kinect data will make the edge sawtooth of this area more obvious, and thus will affect the accuracy of the fingertip detection algorithm afterwards, therefore, after getting the area, apply Bayesian color model to it, remove areas not in the color of the hands and baton, and get the exact area of the baton in hand "Fig. 4".

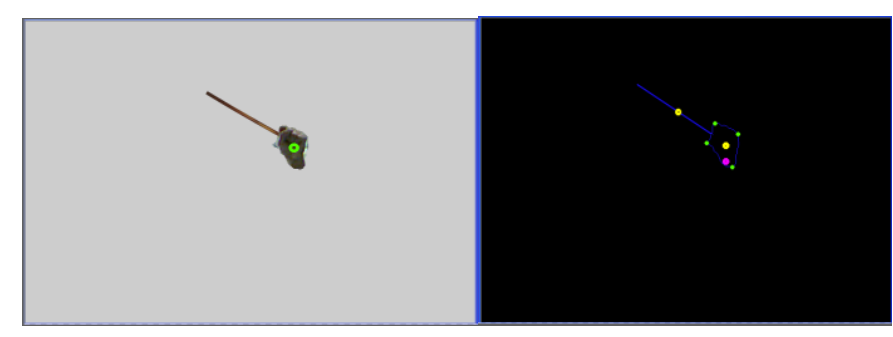

Fig. 4. Motion acquisition image of the baton.

Bayesian color model extracts 20 groups of the user's hand region image within the environment rectangle, and calculates the color threshold of the user's hand region image with the 
relationship between the center point color's mean value and all points within the environment rectangle. And then it uses dichotomy to change the threshold value in order to find out the hand region image value with the minimum noise in the environment rectangle region. Finally, this value is used to binarize the hand and baton posture image [4]. The obtained binarized image is subjected to a characterization process to obtain feature points of the hand region. Through the operation of the feature points, the position information of the hand and baton bone is finally obtained.

\section{RENDER AND OUtPut ANIMATION EFFECT For All BONES}

This module transfers the skeletal information of human body and baton to the graphics rendering engine, renders the picture with the graphics rendering engine, and finally outputs its animation effect "Fig. 5".

As shown in the figure, it can be achieved by dividing into the following steps: (1) the virtual human geometric model creates module. The module consists of building bone part and a bone skin binding part. (2) The motion data captured by Kinect is mapped to the virtual human model by using relocation technology, and then drives the virtual human. (3) The translation compensation mechanism and the distortion processing method module. (4) Output animation by graphic rendering engine.

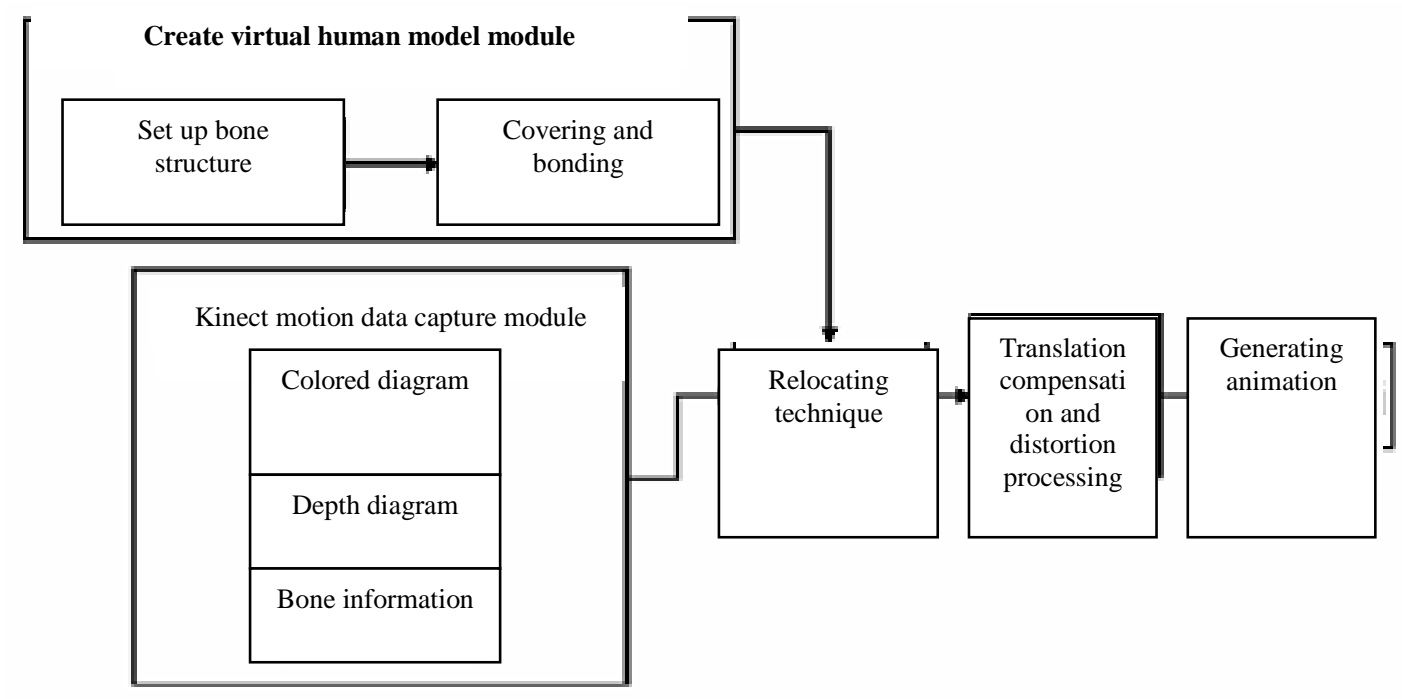

Fig. 5. Output and rendering process of the bone animation.

\section{CONCLUSION}

The remote virtual conducting system connects the performance of the stage presentation with the behavior of the conductor and some performance objects, and introduces the latest technologies such as the interactive presentation combining the virtual and actual to the stage art. It implements positioning and data collection to the various actual performance elements by using the sensor technology, augmented reality technology, human-computer interaction and real-time rendering technology, the stage model established in early phase of creation, and the sensor. Relevant stage representation is triggered by using human-computer interaction, and brand new performance ways different from the past stage art are constantly provided a new, innovating and enriching the original stage performance system. Through the deep research of its key technology and application specification, design and submit relevant stage virtual and actual integrative system to integrate presentation technical specification and related draft standard, make it one of the main technical means to enhance the performance of the stage art. Reduce the creation and rehearsal time in earlier phase, significantly improve work efficiency, enhance creative ability, and provide modern new integrated stage solutions.

\section{REFERENCES}

[1] Han Chunqi.Study on the artistic characteristics ofstage art of dance drama[J] .Journal of Beijing Dance Academy, 2003,03: 76-84.

[2] Wu Yonghai.exploration of application of stereoscopic images to the interactive virtual display [D]. Beijing Institute of Graphic Communication, 2013.

[3] Qin Meng. Design and experience study of somatosensory game motion based on the Peking Opera performance [D]. Harbin Institute of Technology, 2014.

[4] Ren Yafei. Research and realization of interactive media art design system [D]. Hangzhou University of Electronic Science and Technology, 2011. 